\title{
Theileriosis in six dogs in South Africa and its potential clinical significance
}

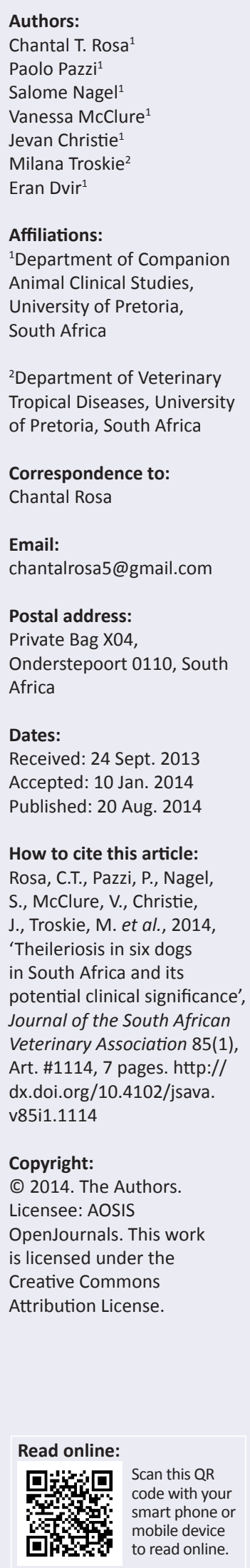

Theileriosis is a tick-borne disease caused by a piroplasma of the genus Theileria that can cause anaemia and thrombocytopenia. Its clinical importance for dogs' remains poorly understood, as only some develop clinical signs. In this study, physical and laboratory findings, treatment and outcomes of six client-owned diseased dogs presented at the Onderstepoort Veterinary Academic Hospital are described retrospectively. In the dogs, Theileria species $(n=4)$ and Theileria equi $(n=2)$ were detected by a polymerase chain reaction (PCR)-reverse blot hybridisation assay in blood samples, whilst PCR for Babesia, Anaplasma and Ehrlichia were negative. The most common physical findings were pale mucous membranes (five out of six dogs), bleeding tendencies (five out of six dogs) and lethargy (three out of six dogs). All dogs were thrombocytopenic [median $59.5 \times 10^{9} / \mathrm{L}$ (range 13-199)] and five out of six dogs were anaemic [median haematocrit 18\% (range 5-32)]. Bone marrow core biopsies performed in two dogs showed myelofibrosis. Theileriosis was treated with imidocarb dipropionate and the suspected secondary immune-mediated haematological disorders with prednisolone and azathioprine. Five dogs achieved clinical cure and post-treatment PCR performed in three out of five dogs confirmed absence of circulating parasitaemia. An immune-mediated response to Theileria species is thought to result in anaemia and/or thrombocytopenia in diseased dogs with theileriosis. A bleeding tendency, most likely secondary to thrombocytopenia and/or thrombocytopathy, was the most significant clinical finding in these cases. The link between thrombocytopenia, anaemia and myelofibrosis in theileriosis requires further investigation and theileriosis should be considered a differential diagnosis for dogs presenting with anaemia and/or thrombocytopenia in endemic tick-borne disease areas.

\section{Introduction}

Theileriosis in dogs may be an emerging tick-borne disease (Chomel 2011), which is caused by piroplasmosis due to organisms of the genus Theileria located within erythrocytes. It can cause anaemia and/or thrombocytopenia secondary to an immune-mediated syndrome (Beck et al. 2009; Fritz 2010; Garcia 2006; Matjila et al. 2008). The size of the pear-shaped intraerythrocytic piroplasma stages evaluated on blood smear examinations has traditionally been used for the identification of Babesia species in dogs, with large forms being associated with Babesia canis and small ones with Babesia gibsoni. However, more sensitive molecular techniques such as polymerase chain reaction (PCR) and deoxyribonucleic acid (DNA) sequencing have further allowed the identification and differentiation of several large and small agents of canine piroplasmosis (Simões et al. 2011). Sequencing and phylogenetic analysis of the ssrRNA gene has allowed reclassification of the canine piroplasms into true Babesia species (B. canis and B. gibsoni), Theileria annae or Babesia microti-like (phylogenetically close to zoonotic B. microti of humans) and Theileria-like groups (Dixit, Dixit \& Varshney 2010). Theileria annae, Theileria equi and Theileria annulata have all been identified in diseased dogs in Spain (Garcia 2006), South Africa (Matjila et al. 2008), Croatia (Beck et al. 2009), France (Fritz 2010) and Portugal (Simões et al. 2011). Dogs have presented with: fever (Fritz 2010; Zahler et al. 2000); anaemia (Beck et al. 2009; Camacho et al. 2002; Fritz 2010; Garcia 2006; Matjila et al. 2008; Zahler et al. 2000); thrombocytopenia (Beck et al. 2009; Fritz 2010; Garcia 2006; Irwin 2010; Matjila et al. 2008; Zahler et al. 2000); immune-mediated syndrome (Fritz 2010; Garcia 2006; Matjila et al. 2008); and renal failure (Garcia 2006).

Many diseased dogs that were reported in previous studies had concurrent haemoparasitosis, therefore an association between theileriosis and the clinical signs could not be established, and treatment and outcomes were also not reported (Beck et al. 2009; Criado-Fornelio et al. 2003; Fritz 2010; Matjila et al. 2008). The pathophysiology of detected Theileria species in dogs remains unknown (Chomel 2011). A chronic established host-parasite commensal relationship or a less virulent parasite relationship compared to the typical host (sable antelopes, horses and cattle) has been proposed (Criado-Fornelio et al. 2003; Matjila et al. 2008), which could explain why some dogs remain subclinically infected. In dogs presenting with anaemia and/ 
or thrombocytopenia, an immune-mediated process has been proposed (Dixit et al. 2010; Fritz 2010; Garcia 2006; Irwin 2010; Matjila et al. 2008; Torina et al. 2007). Coinfections with other vector-born haemoparasites such as Babesia, Ehrlichia, Anaplasma, Bartonella and Rickettsia species can occur (Kordick et al. 1999; Matjila et al. 2008; Yabsley et al. 2008).

Peripheral blood smears can be used to detect the piroplasms within erythrocytes (Dixit et al. 2010), but the genotype identification requires PCR (Irwin 2010; Simões et al. 2011). Polymerase chain reaction has therefore become an essential diagnostic tool for genera and species identification (Ashley, Hackner \& Prittie 2012; Boozer \& Macintire 2005; Criado et al. 2006; Criado-Fornelio et al. 2009; Simões et al. 2011). Imidocarb dipropionate has been assumed to be the drug of choice in dogs and other species (Camacho et al. 2002; Fritz 2010; Simões et al. 2011) based on reported post-treatment clinical improvement and negative PCR (Dixit et al. 2010; Fritz 2010; Gubbels et al. 1999; Simões et al. 2011).

The objectives of this study were to retrospectively describe the clinical manifestations of dogs exclusively diagnosed with theileriosis by PCR, their response to treatment and to alert veterinarians in endemic tick-borne disease areas of the existence of this piroplasmosis in dogs. The speculative pathophysiology of the clinical findings is discussed.

\section{Material and methods}

\section{Animals}

The medical records of client-owned clinically ill dogs presented during 2010-2012 at the Onderstepoort Veterinary Academic Hospital in South Africa with Theileria species detected in blood by PCR were retrospectively reviewed. Inclusion criteria were diseased dogs in which only Theileria species were detected in whole blood by PCR followed by a Reverse Line Blot (RLB) hybridisation assay, whilst negative for other haemoparasitoses such as ehrlichiosis, anaplasmosis, babesiosis or hepatozoonosis. Three additional dogs were excluded due to other concurrent haemoparasites. Signalment, seasonality, presenting complaints, physical examination findings, laboratory test results, imaging findings, treatments and outcomes of all dogs were recorded and compared. A physical examination, peripheral blood smear, urinalysis, faecal analysis and blood collection by jugular venipuncture into ethylenediamine tetra-acetic acid (EDTA) and serum biochemistry tubes were performed at admission.

\section{Laboratory tests}

Central and peripheral blood smears were done at admission to screen for common haemoparasites (Babesia and Hepatozoon species) and to assess changes to erythrocyte morphology, and colour, and platelet count. A complete blood count (CBC) (ADVIA 2120 Haematology System, Siemens, South Africa), in-saline agglutination (ISA) test, and serum biochemistry profile (albumin, globulins, total proteins, glucose, urea, creatinine, cholesterol, alanine aminotransferase, alkaline phosphatase, sodium, potassium, chloride) (Cobas Integra 400 plus, Roche, South Africa) were performed in all dogs. A Coombs' test was performed in three out of six dogs presenting with anaemia at admission. Bone marrow biopsy and cytology were performed in two dogs presenting with non-regenerative anaemia.

\section{Molecular detection}

The DNA was extracted from $200 \mu \mathrm{L}$ of the EDTA-buffered whole blood using the commercially available DNA Kit (QIAamp ${ }^{\circledR}$ DNA Mini Kit, Qiagen, Cape Town, South Africa), according to the manufacturer's instructions. The extracted DNA was eluted in $100 \mu \mathrm{L}$ elution buffer and stored at $4{ }^{\circ} \mathrm{C}$ until analysis. Amplification by PCR of the variable region in the 16S ribosomal RNA gene for Ehrlichia and Anaplasma, and in the 18S ribosomal RNA gene for Theileria and Babesia using specific PCR-primers located within the conserved parts of the rRNA gene of the target organisms was performed. Both PCR-primers sets had matching melting temperatures and thus the same PCR programme could be used for both reactions. The resulting PCR products were hybridised and each species (Ehrlichia, Anaplasma, Theileria, Babesia) identified by a species-specific oligonucleotide probe using a line-blotter apparatus, as follows. The forward primer, RLB-F [5'-GAC ACA GGG AGG TAG TGA CAA G-3'] and the biotin-labelled reverse primer, RLB-R [5'-BiotinCTA AGA ATT TCA CCT CTA ACA GT-3'] were used to amplify the 460-bp - 520-bp fragment of the 18S SSU rRNA gene spanning the V4 hypervariable region of Theileria and Babesia species (Gubbels et al. 1999; Matjila et al. 2004). For the Ehrlichia and Anaplasma, a forward primer Ehr-F (5'-GGA ATT CAG AGT TGG ATC MTG GYT CAG-3') and the reverse primer Ehr-R (5'-biotin-CGG GAT CCC GAG TTT GCC GGG ACT TYT TCT-3') amplifying the fragment of $460 \mathrm{bp}-520 \mathrm{bp}$ from the V1 hypervariable region of the 16S SSU rRNA gene were used (Bekker et al. 2002). A reaction mixture of $25 \mu \mathrm{L}$ of a ready-to-use mixture for high-specificity real-time quantitative DNA amplification (Platinum Quantitative PCR Supermix-UDG, Celtic Molecular Diagnostics, Cape Town, South Africa), 20 pmol $(0.25 \mu \mathrm{L})$ of both forward and reverse primers (Forward and reverse primers, Inqaba Biotec, Pretoria, South Africa), $2.5 \mu \mathrm{L}$ of the extracted DNA template and $9.5 \mu \mathrm{L}$ of molecular grade water was performed in an automated DNA thermal cycler (GeneAmp ${ }^{\circledR}$ PCR System 9700 (automated DNA thermal cycler), Applied Biosystems, Foster City, USA). A touchdown PCR programme was used and the final extension cycle completed the PCR at $72{ }^{\circ} \mathrm{C}$ for seven minutes and was left at $4{ }^{\circ} \mathrm{C}$. The RLB hybridisation was performed using PCR products as described by Gubbels et al. (1999). A plasmid control was used as an internal positive control to ensure that all the Babesia/Theileria and Ehrlichia/Anaplasma speciesspecific oligonucleotides were correctly bound to the RLB membrane and reacting adequately. After stringent washing to remove unbound PCR products, the hybridised PCR products were visualised using chemiluminescence and the microorganisms identified. 


\section{Imaging}

Thoracic radiographs were taken and abdominal ultrasound was performed in all cases to rule out other causes of anaemia and thrombocytopenia.

\section{Treatment}

The veterinarian responsible for each case prescribed medical treatments, apart from imidocarb diproprionate (Forray, imidocarb diproprionate $12 \% \mathrm{~m} / \mathrm{v}$, Intervet Schering-Plough Animal Health SA, Isando, South Africa); these included blood transfusions, prednisolone and azathioprine.

\section{Monitoring}

Complete blood counts in every patient were monitored during hospitalisation and treatment. Follow-up consultations performed in five out of six dogs had complete history, physical examinations and $\mathrm{CBC}$ recorded. The remaining dog was euthanised five days post-admission due to financial constraints. In three out of six dogs, whole blood PCR was repeated between one and three months post-treatment. When improvement and normalisation of the haematocrit and platelet count were documented, clinical cure was considered to have been attained.

\section{Results}

\section{Signalment}

Dogs with theileriosis ranged from three to 10 years old with a median of seven-and-a-half years.

Five dogs were pure breed dogs (Labrador retriever, Beagle, Bullmastiff, Bloodhound and Maltese) and one dog was a small crossbreed. Half of the dogs were males and the other half females.

\section{Seasonality}

Three dogs were diagnosed in winter (June to August), two in spring (September to November) and one in autumn (March to May). No cases were diagnosed in summer (December to February).

\section{Clinical signs and physical abnormalities}

Clinical complaints and physical abnormalities detected at presentation were recorded (Table 1). The most common clinical findings were pale mucous membranes (five out of six dogs); bleeding tendency (five out of six dogs) characterised by oral bleeding, petechiae, ecchymoses, haematuria or haematochezia and lethargy (three out of six dogs).

\section{Laboratory findings Faecal analysis}

On faecal analysis, no intestinal parasites or protozoa were detected. One dog had haematochezia, which was thought to be related to the dog's severe thrombocytopenia $\left(15 \times 10^{9} / \mathrm{L}\right.$; RI 200-500).

\section{Complete blood count}

No haemoparasites were detected on peripheral or central blood smears. All dogs had platelet counts below the reference range [median $59.5 \times 10^{9} / \mathrm{L}$ (range 13-199), reference interval (RI) 200-500] and an increased mean platelet volume [median 17.05 fL (range 12.2-19.6, RI 6-11)] (Table 2). Although all dogs had platelet counts below the RI, the thrombocytopenia in two out of six dogs was mild and clinically insignificant $\left(181 \times 10^{9} / \mathrm{L}\right.$ and $\left.199 \times 10^{9} / \mathrm{L}\right)$. In five out of six dogs concurrent anaemia was detected [median haematocrit $18 \%$ (range 5-2, RI 37-55)]. The anaemia was macrocytic hypochromic regenerative (absolute reticulocyte count above 60) [median absolute reticulocyte count 123.91 (range 98.06-276.00)] in three out of five dogs and normocytic normochromic non-regenerative in two out of five dogs with absolute reticulocyte counts of 17.1 and 19.4. Howell-Jolly bodies were detected in one dog. The dog without anaemia had a haematocrit of $60 \%$ and a severe thrombocytopenia $\left(36 \times 10^{9} / \mathrm{L}\right)$. Two dogs at admission had mild to moderate leucocytosis due to a mature neutrophilia and monocytosis. Median white cell count of all dogs at admission was $8.23 \times 10^{9} /$ L (range 3.27-38.11, RI 6-15).

The anaemia and thrombocytopenia detected at presentation in five out of six dogs normalised after treatment (Table 2). The remaining dog was euthanised five days post-diagnosis. At the end of treatment the haematocrit [median 35\% (range 31-36, RI 37-55)], platelet count [median $630 \times 10^{9} / \mathrm{L}$ (range 314-943, RI 200-500)] and white cell count [median $17.32 \times 10^{9} / \mathrm{L}$ (range 8.32-47.12, RI 6-15)] increased.

\section{In-saline agglutination and Coombs' tests}

In-saline agglutination test (ISA) was positive in one out of six dogs at admission and became negative four days after initiation of treatment. Coombs' test performed in three out of six dogs at admission was negative in all cases.

\section{Serum biochemistry profile}

Most serum biochemistry parameters and electrolytes in all dogs at admission were within the reference range (Table 3).

\section{Urinalysis}

Urinalysis was performed in five out of six dogs at admission. No abnormalities were detected in two out of five dogs,

TABLE 1: Clinical signs in six dogs with theileriosis.

\begin{tabular}{ll}
\hline Clinical signs & Number of dogs $(\boldsymbol{n}=6)$ \\
\hline Pale mucous membranes & 5 \\
Lethargy & 3 \\
Oral bleeding & 3 \\
Splenomegaly & 2 \\
Haemoglobinuria & 2 \\
Haematochezia & 2 \\
Haematuria & 1 \\
Physiologic heart murmur & 2 \\
Inappetence & 2 \\
Petechiae/ecchymoses & 2 \\
Pyrexia & 1 \\
\hline
\end{tabular}


TABLE 2: Description of the haematological parameters at admission and post-treatment of dogs with theileriosis.

\begin{tabular}{|c|c|c|c|c|c|c|c|c|c|}
\hline \multirow[t]{2}{*}{ Parameter } & \multirow[t]{2}{*}{ RI } & \multicolumn{4}{|c|}{ CBC at admission } & \multicolumn{4}{|c|}{ CBC post treatment } \\
\hline & & Median & Range & $\begin{array}{c}n \text { dogs } \\
\text { below RI } \\
(n=6)\end{array}$ & $\begin{array}{c}n \text { dogs } \\
\text { above RI } \\
(n=6)\end{array}$ & Median & Range & $\begin{array}{c}n \text { dogs } \\
\text { below RI } \\
(n=5)\end{array}$ & $\begin{array}{c}n \text { dogs } \\
\text { above RI } \\
(n=5)\end{array}$ \\
\hline Haemoglobin (g/L) & $120.00-180.00$ & 62.50 & $14.00-195.00$ & 5 & 1 & 122.00 & $99.00-137.00$ & 2 & 0 \\
\hline RBC count $\left(x 10^{12} / L\right)$ & $5.50-8.50$ & 2.55 & $0.55-8.83$ & 5 & 1 & 4.67 & $3.75-5.76$ & 4 & 0 \\
\hline Haematocrit (\%) & $37.00-55.00$ & 18.00 & $5.00-60.00$ & 5 & 1 & 35.00 & $31.00-40.00$ & 4 & 0 \\
\hline $\operatorname{MCV}(f L)$ & $60.00-77.00$ & 77.20 & $68.00-96.70$ & 0 & 3 & 74.70 & $69.90-84.40$ & 0 & 0 \\
\hline $\mathrm{MCH}(\mathrm{g} / \mathrm{dL})$ & $21.80-26.00$ & 23.80 & $22.10-27.50$ & 0 & 2 & 25.80 & $23.70-26.50$ & 0 & 0 \\
\hline $\mathrm{MCHC}(\mathrm{g} / \mathrm{dL})$ & $32.00-36.00$ & 31.35 & $27.20-35.10$ & 3 & 0 & 33.90 & $31.40-35.30$ & 0 & 0 \\
\hline RDW (\%) & $13.20-17.40$ & 19.65 & $14.70-26.80$ & 0 & 4 & 15.10 & $14.60-18.20$ & 0 & 2 \\
\hline Reticulocyte count (\%) & $0.50-1.50$ & 3.48 & $1.80-9.08$ & 0 & 5 & - & - & - & - \\
\hline WCC (x109/L) & $6.00-15.00$ & 8.23 & $3.27-38.11$ & 1 & 2 & 17.32 & $8.32-47.12$ & 0 & 4 \\
\hline Mature neutrophils ( $\left.\times 10^{9} / \mathrm{L}\right)$ & $3.00-11.50$ & 5.60 & $2.22-31.63$ & 1 & 2 & 15.59 & $4.58-41.47$ & 0 & 4 \\
\hline Immature neutrophils $\left(\times 10^{9} / L\right)$ & $0.00-0.50$ & 0.47 & $0.00-1.52$ & 0 & 3 & 0.21 & $0.00-2.36$ & 0 & 1 \\
\hline Lymphocytes $\left(\times 10^{9} / L\right)$ & $1.00-4.80$ & 1.61 & $0.50-2.55$ & 2 & 0 & 0.94 & $0.35-1.09$ & 3 & 0 \\
\hline Monocytes (x109/L) & $0.15-1.35$ & 0.76 & $0.20-3.43$ & 0 & 2 & 1.04 & $0.83-2.36$ & 0 & 1 \\
\hline Eosinophils (x109/L) & $0.10-1.25$ & 0.05 & $0.00-0.19$ & 5 & 0 & 0.00 & $0.00-2.08$ & 3 & 1 \\
\hline Basophils $\left(x 10^{9} / L\right)$ & $0.00-0.10$ & 0.00 & $0.00-0.00$ & 0 & 0 & 0.00 & $0.00-0.00$ & 0 & 0 \\
\hline Platelet count (x109/L) & $200.00-500.00$ & 60.00 & $13.00-199.00$ & 6 & 0 & 630.00 & $314.00-943.00$ & 0 & 3 \\
\hline MPV (fL) & $6.00-11.00$ & 17.05 & $12.20-19.60$ & 0 & 6 & 11.00 & $9.00-13.20$ & 0 & 2 \\
\hline Anisocytosis & $0.00-0.00$ & 4.00 & $2.00-5.00$ & - & 6 & 2.00 & $0.00-4.00$ & - & 4 \\
\hline Polychromasia & $0.00-0.00$ & 1.00 & $0.00-3.00$ & - & 4 & 0.00 & $0.00-2.00$ & - & 2 \\
\hline Normoblasts (\%) & $<0.05$ & 5.50 & $0.00-38.00$ & - & 4 & 1.50 & $<0.05-2.00$ & - & 2 \\
\hline Spherocytes (\%) & $<1.00$ & 10.00 & $0.00-70.00$ & - & 4 & $<1.00$ & $<1.00$ & - & 0 \\
\hline ISA & -ve & - & -ve to + & 5 -ve & $1+v e$ & - & -ve & - & 0 \\
\hline ARC & $\begin{array}{l}>60.00 \\
\text { regenerative }\end{array}$ & 98.06 & $17.10-276.00$ & 2 & 3 & - & - & - & - \\
\hline
\end{tabular}

ARC, absolute reticulocyte count; CBC, complete blood count; ISA, in-saline agglutination; $n$, number; $\mathrm{MCH}$, mean corpuscular haemoglobin; $\mathrm{MCHC}$, mean corpuscular haemoglobin concentration $\mathrm{MCV}$, mean corpuscular volume; MPV, mean platelet volume; RBC, red blood cells; RDW, red cell distribution width; RI, reference interval; WCC, white cell count; -ve/+, negative or positive.

whilst the remaining three out of five dogs had inadequately concentrated urine (specific gravity range 1.015-1.016) confirmed by repeated sampling on consecutive days. Additionally, two of these dogs had haemoglobinuria (dogs with haematocrit of $5 \%$ and $8 \%$ ) and one had haematuria (a dog with platelet count of $\left.15 \times 10^{9} / \mathrm{L}\right)$.

\section{Bone marrow evaluation}

Bone marrow cytology and core biopsies performed in two out of six dogs with non-regenerative anaemia revealed hypocellular bone marrow and increased fibrotic tissue deposition compatible with myelofibrosis in both cases. Increased iron deposition was also evident on the bone marrow evaluation of one of the dogs.

\section{Molecular testing}

In two out of six dogs, Theileria equi was identified. In the remaining four out of six dogs, the PCR product did not hybridise to any species-specific probe, only to the Theileria genus-specific catchall probe. Polymerase chain reaction assays for the remaining haemoparasites tested were negative in all dogs.

\section{Imaging}

In five out of six dogs, no radiographic abnormalities were detected. One dog had radiographic signs compatible with spirocercosis, which was considered to be an incidental finding unrelated to the clinical signs. The spirocercosis was thought to be non-neoplastic due to positive response to treatment and the absence of metastasis on thoracic radiographs. Abnormalities detected on abdominal ultrasound included splenomegaly (four out of six dogs) and hepatomegaly (three out of six dogs). Fine-needle aspirates of the enlarged abdominal organs were performed in all dogs and were compatible with extramedullary haematopoiesis.

\section{Treatment and outcome}

Treatment and outcomes were reported in five out of six dogs, as the remaining dog was euthanised five days post-diagnosis due to financial constraints and poor clinical improvement. For treatment of the theileriosis, two injections of imidocarb dipropionate $6 \mathrm{mg} / \mathrm{kg}$ were administered subcutaneously (SC) 14 days apart (Plumb 2008). A transfusion of packed canine red blood cells was administered to four out of six dogs with anaemia at admission after blood and urine collection. Prednisolone and azathioprine were administered at immune-suppressive doses in all dogs for suspected immune-mediated haematological disorders secondary to the theileriosis due to continuous drop of the haematocrit, presence of spherocytosis and anisocytosis, haemoglobinuria (two dogs) and absence of other detectable causes. The dog with non-neoplastic spirocercosis was treated with doramectin $0.4 \mathrm{mg} / \mathrm{kg}$ SC every 14 days for a total of six treatments (Mazaki-Tovi 2002) with a positive response.

Post-treatment complete blood count (Table 2) of five out of six dogs demonstrated clinical cure, with complete resolution of clinical signs and correction of the anaemia and/or thrombocytopenia. Post-treatment blood PCR performed in 
three out of five dogs was negative for all haemoparasites, therefore also supportive of clinical cure.

\section{Discussion}

This retrospective study describes the common findings in diseased dogs with Theileria species detected by PCR-reverse blot hybridisation assay, which included pale mucous membranes, bleeding tendencies and lethargy. All dogs were thrombocytopenic and five out of six were anaemic. Bone marrow core biopsies performed in two dogs showed myelofibrosis. All treated dogs (five out of six) achieved clinical cure.

The number of reported cases of theileriosis in dogs seems to be increasing (Dixit et al. 2010), with the majority of cases described in the Mediterranean countries (Beck et al. 2009; Fritz 2010; Garcia 2006; Simões et al. 2011) and South Africa. This emphasises the need to better understand the significance of this piroplasma in dogs and to alert veterinarians to its existence. However, the increased number of reported cases could simply relate to the new molecular techniques used in the clinical investigations of diseases.

Blood smear examinations by light microscopy seem to be reasonably sensitive for diagnosis of intraerythrocytic piroplasms (Irwin 2010; Simões \& Waner 2011), but the species or genotype of the organism cannot be determined by morphology alone; they require PCR and genomic sequence analysis (Criado-Fornelio et al. 2003; Harrus \& Waner 2011; Irwin 2010; Matjila et al. 2008; McCullough 2003; Otranto et al. 2010; Simões et al. 2011). In contrast, the detection of chronic and subclinical piroplasmosis in carrier dogs requires molecular tools (serology and PCR) because the sensitivity of microscopy in such cases is very low (Irwin 2010; Matjila et al. 2008), which is most likely the case in the dogs of this study.

Several Theileria species have been identified in both diseased and subclinically infected dogs. Species found in diseased dogs included: Theileria equi in dogs in Southern Europe (Criado-Fornelio et al. 2003), France (Fritz 2010) and Croatia (Beck et al. 2009); Theileria annae in dogs from Germany (Zahler et al. 2000), Spain (Camacho et al. 2002; Garcia 2006),
Croatia (Beck et al. 2009) and Portugal (Simões et al. 2011); and Theileria sable in dogs from South Africa (Matjila et al. 2008). Theileria annulata and T. equi have been identified in subclinically infected dogs in southern Europe (Criado et al. 2006; Criado-Fornelio et al. 2003) and T. annae in a subclinically infected dog in Croatia (Beck et al. 2009). This shows that certain Theileria species might be associated with either a non-clinical state related to a commensal relationship, a less virulent parasite-host relationship (Criado-Fornelio et al. 2003; Matjila et al. 2008), or clinical disease of different severities in dogs (Garcia 2006; Matjila et al. 2008). The specific pathogenic species, treatment and outcomes of canine theileriosis have been poorly described.

The present study only evaluated diseased dogs with theileriosis detected by PCR. Theileria equi was identified in two out of six dogs and Theileria spp. in the remaining four. The pathophysiology of detected Theileria species in dogs remains poorly understood (Dixit et al. 2010; Solano-Gallego \& Baneth 2011) but it seems that haemolytic anaemia, thrombocytopenia, splenomegaly and an immune-mediated syndrome may be associated with this organism (Camacho et al. 2003; Dixit et al. 2010; Fritz 2010; Garcia 2006; Matjila et al. 2008; Simões et al. 2011), as was also seen in the present study. It appears that theileriosis in dogs shares common features with other haemoparasitoses such as babesiosis and ehrlichiosis, namely anaemia and/or thrombocytopenia being hallmarks of all three tick-borne diseases (Ashley et al. 2010; Garcia 2006; Harrus \& Waner 2011; Solano-Galego \& Baneth 2011). Therefore, for the correct treatment and prognosis accurate detection of species and subspecies is important (Dixit et al. 2010).

The thrombocytopenia detected in this study, as in others (Matjila et al. 2008; Simões et al. 2011), was likely to be secondary to an immune-mediated destruction, splenic sequestration or platelet consumption. It was considered the most likely cause of the bleeding tendency noted in five out of six dogs, characterised by the presence of oral bleeding, petechiae and ecchymoses. A concurrent thrombocytopathy, as described in canine ehrlichiosis (Harrus, Bark \& Waner 1997; Harrus, Kass, Klement \& Waner 1997; Harrus \& Waner 2011; Little 2010; Matjila et al. 2008; McCullough 2003; Shipov

TABLE 3: Description of the biochemistry profile at admission of dogs diagnosed with canine theileriosis.

\begin{tabular}{|c|c|c|c|c|c|c|}
\hline Parameter & RI & Range & Median & $n$ dogs & $n$ dogs below RI & $n$ dogs above RI \\
\hline $\mathrm{TP}(\mathrm{g} / \mathrm{L})$ & $53.00-75.00$ & $49.70-80.40$ & 60.30 & 6 & 2 & 1 \\
\hline Albumin (g/L) & $27.00-35.00$ & $22.40-35.70$ & 29.55 & 6 & 1 & 1 \\
\hline Globulins (g/L) & $20.00-37.00$ & $20.10-50.90$ & 33.05 & 6 & 0 & 1 \\
\hline $\operatorname{ALT}(U / L)$ & $9.00-73.00$ & $15.00-232.00$ & 94.00 & 4 & 0 & 2 \\
\hline $\operatorname{ALP}(U / L)$ & $65.00-311.00$ & $22.00-144.00$ & 109.00 & 4 & 1 & 0 \\
\hline Urea (mmol/L) & $3.60-8.90$ & $3.30-9.70$ & 5.90 & 4 & 1 & 1 \\
\hline Cholesterol (mmol/L) & $2.70-7.00$ & $3.25-5.60$ & 4.43 & 3 & 0 & 0 \\
\hline Glucose (mmol/L) & $3.30-5.50$ & $4.20-4.60$ & 4.40 & 2 & 0 & 0 \\
\hline Sodium (mmol/L) & $140.00-155.00$ & $143.00-146.20$ & 143.80 & 3 & 0 & 0 \\
\hline Potassium (mmol/L) & $3.60-5.10$ & $3.87-4.84$ & 4.34 & 5 & 0 & 0 \\
\hline
\end{tabular}

ALT, alanine aminotransferase; ALP, alkaline phosphatase; $n$, number; RI, reference interval; TP, total proteins. 
et al. 2008), could also be suggested as a cause of the bleeding tendency; platelet function testing would be required to prove this association. A transient thrombocytopenia due to splenic platelet sequestration and immune-mediated destruction as occurs in B. canis subspecies rossi and B. gibsoni infections (Ashley et al. 2010; Taboada \& Lobetti 2006) could also be the cause of the thrombocytopenia seen in theileriosis. Further studies are required to determine possible links between thrombocytopenia, anaemia and the bone marrow myeloproliferative disorder that were detected in the dogs with theileriosis.

Garcia (2006) found that $90 \%$ of dogs with theileriosis had regenerative anaemia with haematocrits below 31\%, and $75 \%$ of dogs had platelet counts below $75 \times 10^{9} / \mathrm{mL}$ and $50 \%$ below $23 \times 10^{9} / \mathrm{mL}$. All dogs in this study had platelet counts below the RI, with four out of six having a severe thrombocytopenia (median platelet count $59.5 \times 10^{9} / \mathrm{L}$ ). Five dogs had anaemia (median haematocrit 18\%) similar to previous reports (Beck et al. 2009; Fritz 2010; Garcia 2006; Matjila et al. 2008; Simões et al. 2011). The anaemia detected was considered to be either immune-mediated or related to direct parasite-induced injury and/or oxidative injury from haemolytic toxins, as described in canine babesiosis (Harrus, Bark \& Waner 1997; Irwin 2010; Jacobson 2006; McCullough 2003). The presence of anisocytosis and spherocytosis, continual drop of the haematocrit and response to immunesuppressive drugs supports an immune-mediated process, even in the presence of a negative ISA and Coombs' test, as they have low sensitivity. In this study, two out of six dogs had non-regenerative anaemia, contrary to previous studies consistently reporting regenerative anaemia. This lack of regeneration is proposed to be secondary to an immunemediated destruction of bone marrow precursor cells, leading to myelofibrosis and haemosiderin deposition (Hoff et al. 1991; Weiss 2008; Weiss \& Smith 2002), as detected in these two dogs or related to a theileriosis-associated bone marrow suppression, similar to what occurs in the chronic phase of ehrlichiosis (Harrus, Kass, Klement \& Waner 1997, Harrus \& Waner 2011; Little 2010; McCullough 2003; SolanoGallego \& Baneth 2011). The chronicity of infection can be considered a possible determinant factor for bone marrow involvement leading to these different clinicopathological findings observed in theileriosis in dogs.

An immune-mediated glomerulonephropathy with renal azotaemia was described in 36\% of dogs diagnosed with T. annae in Spain (Garcia 2006), a feature not evident in the present study. This is possibly due to a different infecting species and immune-response, or a more chronic subclinical disease or low parasitaemia.

Studies regarding the optimal treatment of canine theileriosis are lacking. Imidocarb dipropionate has been assumed to be the drug of choice at $6 \mathrm{mg} / \mathrm{kg}$ administered SC and repeated 14 days later, for elimination of this parasite (Camacho et al.
2002; Fritz 2010; Simões et al. 2011), as administered in all dogs in this study. All dogs in the current study that received both SC injections of imidocarb diproprionate achieved clinical cure, suggesting that imidocarb dipropionate is efficient in sterilising theileriosis infections in dogs. This is further supported by the negative PCR results after treatment, as suggested in babesiosis (Boozer \& Macintire 2005). To assure parasitological cure, other body tissues such as the spleen, liver and bone marrow should undergo molecular testing for the presence of the parasite.

The relationship between parasite species and clinical signs, morphological characteristics and drug trials against Theileria infection in dogs deserves further investigation. Movements of dogs can expand the geographical distribution of established piroplasms (Irwin 2010), thus, beyond accessible and accurate diagnostic tools, safer and more efficacious drugs against the parasite are necessary.

Limitations of this study included: a small number of dogs; lack of tissue sample confirmation of parasite cure in all dogs; absent follow-up PCR post-treatment in two dogs; lack of confirmation or exclusion of other haemoparasites beyond Babesia sp., Ehrlichia sp., Anaplasma sp. and Hepatozoon canis that could have potentially caused the anaemia and/or thrombocytopaenia; and lack of DNA sequencing in order to clearly define the infecting Theileria agents. Another limitation was the inclusion of a dog with concurrent nonneoplastic spirocercosis in this study, but the authors believe that the spirocercosis was an incidental finding unrelated to the severe macrocytic hypochromic anaemia (haematocrit 5\%, RI 37-55) or the insignificant thrombocytopenia (platelet count $199 \times 10^{9} / \mathrm{L}$, RI 200-400) on presentation, as non-neoplastic spirocercosis is not associated with severe anaemia. Normocytic mild to moderate anaemia is more commonly seen in neoplastic spirocercosis in dogs (Dvir et al. 2008).

Further studies are required to determine the possible links between thrombocytopenia, anaemia and myeloproliferative disorders, as observed in some cases with theileriosis. Further studies are warranted to elucidate the specific pathogenic species and their virulence, in order to better understand and anticipate the clinical manifestations of theileriosis in dogs.

\section{Conclusion}

Veterinarians should be aware of this tick-borne disease and should consider theileriosis as a differential diagnosis for any dog with thrombocytopenia and/or anaemia in an endemic tick-borne disease area. Diseased dogs with a history of exposure to ticks or previous travel to a tick-endemic area should prompt specific investigation for haemoparasitosis, including theileriosis.

Theileria species detected in diseased dogs in South Africa seem to carry a fair-to-good prognosis if early diagnosis, 
adequate treatment with imidocarb dipropionate and symptomatic care are instituted. The pathophysiology of theileriosis is speculated to be immune-mediated leading to thrombocytopenia and anaemia. An immune-mediated bone marrow involvement is also suspected in more severe and chronic cases.

\section{Acknowledgement Competing interests}

The authors declare that they have no financial or personal relationship(s) which may have inappropriately influenced them in writing this article.

\section{Authors' contributions}

C.T.R. (University of Pretoria) is the main author and responsible for the project design and for a clinical case. P.P. (University of Pretoria), S.N. (University of Pretoria), V.M. (University of Pretoria) and J.C. (University of Pretoria) were responsible for the clinical cases, data collection and analysis, and made conceptual contributions. M.T. (University of Pretoria) performed, interpreted the PCR results and described the PCR technique. E.D. (University of Pretoria) was the project leader making fundamental contributions.

\section{References}

Ashley, L.A., Hackner, S.G. \& Prittie, J., 2012, 'Clinical management of canine babesiosis', Journal of Veterinary Emergency and Critical Care 20(1), 77-89.

Beck, R., Vojta, L., Mrljak, V., Marinculic, A., Beck, A., Zivicnjak, T. \& Cacciò, S.M., 2009, 'Diversity of Babesia and Theileria species in symptomatic and asymptomatic dogs in Croatia', International Journal for Parasitology 39, 843-848. http://dx.doi. org/10.1016/j.ijpara.2008.12.005

Bekker, C.P.J., De Vos, S., Taoufik, A., Sparagano, O.A.E. \& Jongejan, F., 2002 'Simultaneous detection of Anaplasma and Ehrlichia species in ruminants and
detection of Ehrlichia ruminantium in Amblyomma variegatum ticks by reverse line blot hybridization', Veterinary Microbiology, $89223-238$. http://dx.doi. org/10.1016/S0378-1135(02)00179-7

Boozer, L. \& Macintire, D., 2005, 'Babesia gibsoni: An emerging pathogen in dogs', Compendium on Continuing Education for the Practicing Veterinarian 27, 33-41.

Camacho, A.T., Pallas, E., Gestal, J.J., Guitian, F.J. \& Olmeda, A.S., 2002, 'Natural infection by a Babesia microti-like piroplasm in a splenectomised dog', Veterinary Record 150, 381-382.

Camacho, A.T., Pallas, E., Gestal, J.J., Guitian, F.J., Olmeda, A.S., Telford, S.R. III \& Spielman, A., 2003, 'Ixodes hexagonous is the main candidate as vector of Theileria annae in Northwest Spain', Veterinary Parasitology, 112, 157-163. http://dx.doi. org/10.1016/S0304-4017(02)00417-X

Chomel, B., 2011, 'Tickborne infections in dogs - an emerging infectious threat', Veterinary Parasitology 179, 294-301. http://dx.doi.org/10.1016/j. vetpar.2011.03.040

Criado, A., Martinez, J., Buling, A., Barba, J.C., Merino, S., Jefferies, R. \& Irwin, P.J., 2006 , 'New data on epizootiology and genetics of piroplasms based on sequences of small ribosomal subunit and cytochrome b genes', Veterinary Parasitology 142 238-247. http://dx.doi.org/10.1016/j.vetpar.2006.07.004

Criado-Fornelio, A., Buling, A., Pingret, J.L., Etievant, M., Boucraut-Baralon, C., Alongi, A., Agnone, A. \& Torina, A., 2009, 'Hemoprotozoa of domestic animals in France: Prevalence and molecular characterization', Veterinary Parasitology 159, 73-76. http://dx.doi.org/10.1016/j.vetpar.2008.10.012

Criado-Fornelio, A., Martinez-Marcos, A., Buling-Saraña, A. \& Barba-Carretero, J.C., 2003, 'Molecular studies on Babesia, Theileria and Hepatozoon in Southern Europe Part I. Epizootiological aspects', Veterinary Parasitology 113, 189-201. http://dx.doi.org/10.1016/S0304-4017(03)00078-5

Dixit, P., Dixit, A.K. \& Varshney, J.P., 2010, 'Evidence of new pathogenic Theileria species in dogs', Journal of Parasitic Diseases 34, 29-32. http://dx.doi.org/10.1007/s12639010-0009-0

Dvir, E., Kirberger, R.M., Mukorera, V., van der Merwe, L.L. \& Clift, S.J., 2008, 'Clinical differentiation between dogs with benign and malignant spirocercosis', Veterinary Parasitology 155, 80-88. http://dx.doi.org/10.1016/j.vetpar.2008.04.006
Fritz, D., 2010, 'A PCR study of piroplasms in 166 dogs and 111 horses in France (March 2006 to March 2008)', Parasitology Research 106, 1339-1342. http://dx.doi. org/10.1007/s00436-010-1804-3

Garcia, A.T.C., 2006, 'Piroplasma infection in dogs in northern Spain', Veterinary Parasitology 138, 97-102. http://dx.doi.org/10.1016/j.vetpar.2006.01.043

Gubbels, J.M., De Vos, A.P., Van der Weide, M., Viseras, J., Schouls, L.M., De Vries, E. \& Jongejan, F., 1999, 'Simultaneous detection of bovine Theileria and Babesia species by reverse line blot hybridization', Journal of Clinical Microbiology 37, 1782-1789.

Harrus, S., Bark, H. \& Waner, T. 1997, 'Canine monocytic ehrlichiosis: An update', Compendium on Continuing Education for the Practicing Veterinarian 19, 431-444.

Harrus, S., Kass, P.H., Klement, E. \& Waner, T., 1997, 'Canine monocytic ehrlichiosis: A retrospective study of 100 cases, and an epidemiological investigation of prognostic indicators for the disease', Veterinary Record 141, 360-363. http://dx.doi. org/10.1136/vr.141.14.360

Harrus, S. \& Waner, T., 2011, 'Diagnosis of canine monocytotropic ehrlichiosis (Ehrlichia canis): An overview', Veterinary Journal 187, 292-296. http://dx.doi.org/10.1016/j. tvjl.2010.02.001

Hoff, B., Lumsden, J.H., Valli, V.E.O. \& Kruth, S.A., 1991, 'Myelofibrosis: Review of clinical and pathological features in fourteen dogs', Canadian Veterinary Journal 32, 357-361.

Irwin, P.J., 2010, 'Canine babesiosis', Veterinary Clinics of North America Small Animal Practice 40, 1141-1156. http://dx.doi.org/10.1016/j.cvsm.2010.08.001

Jacobson, L.S., 2006, 'The South African form of severe and complicated canine babesiosis: Clinical advances 1994-2004', Veterinary Parasitology 138, 126-139. http://dx.doi. org/10.1016/j.vetpar.2006.01.047

Kordick, S.K., Breitschwerdt, E.B., Hegarty, B.C., Southwick, K.L., Colitz, C.M., Hancock, S.I, Bradley, J.M., Rumbough, R., Mcpherson, J.T. \& MacCormack, J.N., 1999, 'Co-infection Bradley, J.M., Rumbough, R., Mcpherson, J.T. \& MacCormack, J.N., 1999, 'Co-infection
with multiple tick-borne pathogens in a Walker Hound kennel in North Carolina', with multiple tick-borne pathogens in a Walker
Journal of Clinical Microbiology 37, 2631-2638.

Little, S.E., 2010, 'Ehrlichiosis and anaplasmosis in dogs and cats', Veterinary Clinics of North America Small Animal Practice 40, 1121-1140. http://dx.doi.org/10.1016/j. cvsm.2010.07.004

Matjila, T.P., Penzhorn, B.L., Bekker, C.P., Nijhof, A.M. \& Jongejan, F., 2004, 'Confirmation of occurrence of Babesia canis vogeli in domestic dogs in South Africa', Veterinary Parasitology 122, 119-125. http://dx.doi.org/10.1016/j.vetpar.2004.03.019

Matjila, P.T., Leisewitz, A.L., Oosthuizen, M.C., Jongejan, F. \& Penzhorn, B,L., 2008 'Detection of Theileria species in dogs in South Africa', Veterinary Parasitology 157 34-40. http://dx.doi.org/10.1016/j.vetpar.2008.06.025

Mazaki-Tovi, M., Baneth, G., Aroch, I., Harrus, S., Kass, P.H., Ben-Ari, T., Zur, G., Aizenberg I., Bark, H. \& Lavy, E., 2002, 'Canine spirocercosis: Clinical, diagnostic, pathologic, and epidemiologic characteristics', Veterinary Parasitology 107, 235-250. http://dx.doi. org/10.1016/S0304-4017(02)00118-8

McCullough, S., 2003, IImmune-mediated hemolytic anaemia: Understanding the nemesis', Veterinary Clinics of North America Small Animal Practice 33, 1295-1315. http://dx.doi.org/10.1016/j.cvsm.2003.08.003

Otranto, D., Testini, G., Dantas-Torres, F., Latrofa, M.S., Vissotto de Paiva Diniz, P.P., de Caprariis, D., Lia, R.P., Mencke, N., Stanneck, D., Capelli, G. \& Breitschwerdt, E.B., 2010, 'Diagnosis of canine vector-borne diseases in young dogs: A longitudinal study', Journal of Clinical Microbiology 48, 3316-3324. http://dx.doi.org/10.1128/ JCM.00379-10

Plumb, D.C., 2008, 'Imidocarb dipropionate', in D.C. Plumb (ed.), Plumb's Veterinary Drug Handbook, 6th edn., pp. 473-474, Wiley-Blackwell, Ames.

Simões, P.B., Cardoso L., Araújo, M., Yisaschar-Mekuzas, Y. \& Baneth, G., 2011, 'Babesiosis due to the canine Babesia microti-like small piroplasm in dogs - first report from Portugal and possible vertical transmission', Parasites and Vectors 4, 50. http://dx.doi. org/10.1186/1756-3305-4-50

Shipov, A., Klement, E., Reuveni-Tager, L., Waner, T. \& Harrus, S., 2008, 'Prognostic indicators for canine monocytic ehrlichiosis', Veterinary Parasitology 153, 131-138. http://dx.doi.org/10.1016/j.vetpar.2008.01.009

Solano-Gallego, L. \& Baneth, G., 2011, 'Babesiosis in dogs and cats - expanding parasitological and clinical spectra', Veterinary Parasitology, 181, 48-60. http://dx.doi org/10.1016/j.vetpar.2011.04.023

Taboada, J. \& Lobetti, R., 2006, 'Babesiosis', in C. Greene (ed.), Infectious Diseases of the Dog and Cat, 3rd edn., pp. 722-735, Elsevier Health Sciences, St Louis.

Torina, A., Vicente, J., Alongi, A., Scimeca, S., Turla, R., Nicosia, S., Di Marco, V., Caracappa, S. \& De la Fuente, J., 2007, 'Observed prevalence of tick-borne pathogens in domestic animals in Sicily, Italy during 2003-2005', Zoonoses and Public Health 54, 8-15. http:// dx.doi.org/10.1111/j.1863-2378.2007.00989.x

Weiss, DJ., 2008, 'Bone marrow pathology in dogs and cats with non-regenerative immunemediated haemolytic anaemia and pure red cell aplasia', Journal of Comparative Pathology 138, 46-53. http://dx.doi.org/10.1016/j.jcpa.2007.10.001

Weiss, D.J. \& Smith, S.A., 2002, 'A retrospective study of 19 cases of canine myelofibrosis', Journal of Veterinary Internal Medicine 16, 174-178. http://dx.doi. org/10.1111/j.1939-1676.2002.tb02351.x

Yabsley, M.J., McKibben, J., Macpherson, C.N., Cattan, P.F., Cherry, N.A., Hegarty, B.C., Breitschwerdt, E.B., O'Connor, T, Chandrashekar, R., Paterson, T., Perea, M.L., Ball, G., Friesen, S., Goedde, J., Henderson, B. \& Sylvester, W., 2008, 'Prevalence of Ehrlichia canis, Anaplasma platys, Babesia canis vogeli, Hepatozoon canis, Bartonella vinsoni canis, Anaplasma platys, Babesia canis vogell, Hepatozoon canis, Bartonella vinsoni 285. http://dx.doi.org/10.1016/j.vetpar.2007.11.008

Zahler, M., Rinder, H., Schein, E. \& Gothe, R., 2000, 'Detection of a new pathogenic Babesia microti-like species in dogs', Veterinary Parasitology 89, 241-248. http://dx.doi. org/10.1016/S0304-4017(00)00202-8 